\title{
On the selection of wind turbine generator based on ARMA time series
}

\begin{abstract}
By increasing the global warming and environmental challenges the industrial countries commit to reduce the gas emissions and develop the renewable energy based on the Kyoto protocol and also renewable portfolio standard (RPS). Among them, wind resources are more important because of the progress in the technology of its facilities to convert wind to electricity. Wind turbines manufacturer companies improve the efficiency of their facilities during the last decade. On the other hand, the wind generator firms and investors intend to select a suitable wind turbine generators (WTGs) based on the wind distribution in a specific region. In this study, Output power (MW) of four different types of WTGs has been compared. The wind speed data has been collected for 10 years from 2000 to 2009 from Canada Swift Current region. Then, the model of wind speed has been predicted with ARMA time series. In the next stage, the output power of each WTG has been determined with probability method. Finally, the suitable WTG is recommended according to the output power produced.
\end{abstract}

Keyword: Renewable energy; WTG; ARMA time series 